\title{
On Periodic solutions for a reduction of Benney chain
}

\author{
Misha Bialy
}

\begin{abstract}
We study periodic solutions for a quasi-linear system, which naturally arises in search of integrable Hamiltonian systems of the form $H=$ $p^{2} / 2+u(q, t)$. Our main result classifies completely periodic solutions for such a 3 by 3 system. We prove that the only periodic solutions have the form of traveling waves so, in particular, the potential $u$ is a function of a linear combination of $t$ and $q$. This result implies that the there are no nontrivial cases of the existence of a fourth power integral of motion for $H$ : if it exists, then it is equal necessarily to the square of a quadratic integral. Our main observation for the quasi-linear system is the genuine non-linearity of the maximal and minimal eigenvalues in the sense of Lax. We use this observation in the hyperbolic region, while the "elliptic" region is treated using the maximum principle.
\end{abstract}

Mathematics Subject Classification (2000). 35L65, 35L67, 70H06.

Keywords. Riemann invariants, Genuine nonlinearity, Rich systems, Benney chain.

\section{Introduction}

Let $H=p^{2} / 2+u(q, t)$ be a Hamiltonian function of a system with 1,5 -degrees of freedom and the potential which is assumed throughout this paper to be a periodic function in both variables. There is a conjecture attributed to Birkhoff saying that the only integrable plane convex billiards are ellipses. The direct analog of this conjecture for the Hamiltonian system with 1,5-degrees of freedom would be the claim that the only integrable Hamiltonian functions of the form $H=p^{2} / 2+u(q, t)$ are those having potential functions $u$ which are periodic functions of the form $u=u(m q+n t)$. There have been several attempts to approach

This paper was started at the Island 3 meeting on July 2007. It is my pleasure to thank the organizers for their support. 
this problem. Let me refer to the works $[1,2,4,6,12]$ for various approaches to this circle of questions which remain outside the discussion of this paper. Let me mention that it is a well-known fact, that for general potential functions $u(q, t)$ the Hamiltonian system cannot have additional integrals. This is a consequence of complicated dynamics for such a system in general: non-existence of invariant tori, chaotic regions and so on. So in this sense the paper is devoted to the classification of an exceptional event of integrability. Our goal is to push forward an unexpected relation of integrability with the existence of smooth periodic solutions for a remarkable quasi-linear system (2).

In the present note we restrict this question to the search for additional integrals which are polynomial with respect to the momenta variable $p$ with coefficients which are periodic functions of $q$ and $t$ (in mechanics, for some mysterious reason, integrals always appear to be polynomial in momenta variables). More precisely we want to find all those potential functions $u(q, t)$ for which there exists an additional function $F(p, q, t)$ invariant under the Hamiltonian flow (such an $F$ is called the first integral of motion) which is a polynomial in the variable $p$ of a given degree, say $n+1$, having all the coefficients periodic in $q$ and $t$. Write

$$
F(p, q, t)=u_{-1} p^{n+1}+u_{0} p^{n}+u_{1} p^{n-1}+\cdots+u_{n}
$$

and substitute into the equation of conservation of $F$.

$$
F_{t}+p F_{q}-u_{q} F_{p}=0
$$

Equating to zero the coefficients of various powers of $p$, one easily obtains the following information. The coefficient $u_{-1}$ must be a constant, which will be normalized to be $\frac{1}{n+1}$. Also $u_{0}$ must be a constant, which we shall assume to be zero (this can be achieved by a linear change of coordinates on the configuration space $\left.\mathbb{T}^{2}\right)$. Moreover the coefficient $u_{1}$ satisfies $\left(u_{1}\right)_{q}=(u)_{q}$. Therefore, $u_{1}$ and the potential $u$ will be assumed to be equal (the addition of any function of $t$ to the potential $u$ does not change the Hamiltonian equations). Moreover, the column of the rest of the coefficients $U=\left(u_{1}, \ldots, u_{n}\right)^{t}$ satisfies the following quasi-linear system of equations.

$$
U_{t}+A(U) U_{q}=0, \quad A(U)=-\left(\begin{array}{cccccc}
0 & -1 & 0 & \cdots & 0 & 0 \\
(n-1) u_{1} & 0 & -1 & 0 & \cdots & 0 \\
\vdots & \vdots & \vdots & \vdots & \vdots & \vdots \\
2 u_{n-2} & 0 & 0 & \cdots & 0 & -1 \\
u_{n-1} & 0 & 0 & \cdots & 0 & 0
\end{array}\right)
$$

Notice that the derivative $F_{p}$ of $F$ coincides with the characteristic polynomial of $A(U)$.

Our main result is the following classification of smooth periodic solutions for the system.

Theorem 1.1. Let $n=3$. Then the only periodic solution of the quasi-linear system (2) is the standing wave solution, where $u_{1}, u_{2}, u_{3}$ do not depend on $t$. 
Corollary 1.2. Let $F=1 / 4 p^{4}+u_{1} p^{2}+u_{2} p+u_{3}$ be a polynomial of degree four with periodic coefficients which satisfies the Eq. (1). Then there necessarily exists a quadratic integral, i.e. the energy $H$, and $F$ is a function of $H$.

In fact, the system (2) is well known in the integrable systems community. This is the so called dispersionless Lax reduction of the moments Benney chain (see for example $[8,9,11,14]$ and references therein) arrising in the theory of long shallow water waves. There are many beautiful properties of this system. Most important for this paper is the property of the system (2) to be rich (or semi-hamiltonian) (see [13]). This means, by definition, that (2) can be written as a system of conservation laws and in addition the system is diagonalizable, i.e. it has a diagonal form in some coordinates, called Riemann invariants (see (3) below). ${ }^{1}$ It follows by a very general argument that the eigenvalues of any rich system must satisfy certain compatibility relations (see [13], Theorem 12.1.1 and also Corollary 2.3 below). These relations on eigenvalues provide certain exactness for the system and so the original Lax analysis applies, exactly in the same manner as it was done by Lax in [10] for 2 by 2 systems (see [13], p.146 and below). The original Lax method relies heavily on the possibility to write the 2 by 2 system in diagonal form, and also on the genuine non-linearity of the eigenvalues. Lax analysis was used in [3] for a 2 by 2 system of the form (2) where it was proved that the only periodic solutions for that case are constants. For rich systems of the greater size the original method by Lax applies, if the eigenvalues are known to be genuinely nonlinear. We shall prove below (Corollary 2.2) that in the strictly Hyperbolic region maximal and minimal eigenvalues for our system are in fact genuinely nonlinear (this property can be explained geometrically, but we prove it by direct computation in Lemma 2.1 below).

Notice, that it is not known a priori that the periodic solution in question of the system (2) lies entirely in the Hyperbolic region. Therefore, in order to prove the main theorem we distinguish between different regions: the strictly Hyperbolic region $\Omega_{h}$ where all three eigenvalues of $A(U)$ are real and distinct, the "Elliptic" region $\Omega_{e}$, where two eigenvalues are complex conjugate and the third one is real, and finally the region of Degeneracy $\Omega_{0}$, where at least two of the eigenvalues collide. It turns out that in all these regions the behavior of solutions can be understood completely. In the strictly Hyperbolic region we use genuine nonlinearity while in the Elliptic region we use strong maximum principle. The proof of Theorem 1.1 is obtained just by patching together the information of all the cases.

Organization of the paper is as follows. In the next Section we shall explain the two basic observations mentioned above concerning the hyperbolic situation. In Sects. 3, 4, 5 we study the regions $\Omega_{h}, \Omega_{e}, \Omega_{0}$, respectively for the case of $n=3$. In Sect. 6 we prove formulas for derivatives of the eigenvalues and verify, for the sake of completeness, the compatibility conditions of Rich systems.

\footnotetext{
${ }^{1}$ I was told by Pavlov that this is a known fact among the specialists in integrable systems, see also [5] where it was rediscovered.
} 


\section{Main observations}

Let me denote by $\lambda_{1}, \ldots, \lambda_{n}$ the roots of the polynomial $F_{p}$. And let $r_{i}=F\left(\lambda_{i}\right)$ be the corresponding critical values. The starting point for us is a beautiful classical theorem by MacLane, stating that the mapping $\left(u_{1} \ldots u_{n}\right) \mapsto\left(r_{1}, \ldots, r_{n}\right)$ is in fact a global diffeomorphism between the domain of strict Hyperbolicity (that is the domain of all $U=\left(u_{1}, \ldots, u_{n}\right)^{t}$ where all the roots of the polynomial $F_{p}$ are real and distinct) and the domain of all possible critical values in $\mathbb{R}^{n}$ that is of all those $\left(r_{1}, \ldots, r_{n}\right)$ such that the differences $\left(r_{k}-r_{k+1}\right)$ have the sign $(-1)^{k+n}$ for all $k=1, \ldots, n-1$ (we refer to [7] for Vinberg's proof of this theorem and further results and discussions). According to this theorem $\left(r_{1}, \ldots, r_{n}\right)$ can be taken as regular global coordinates in this domain.

The importance of these coordinates for our system becomes clear from the following computation. Substitute $p=\lambda_{i}$ into the Eq. (1). One gets the following diagonal system on the variables $r_{i}$ (they are called Riemann invariants)

$$
\left(r_{i}\right)_{t}+\lambda_{i}\left(r_{1}, \ldots, r_{n}\right)\left(r_{i}\right)_{q}=0, \quad i=1, \ldots, n .
$$

The derivatives of the roots $\lambda_{i}$ with respect to the critical values $r_{i}$ satisfy the following relations.

Lemma 2.1. The following formulas hold true:

(a) $\partial_{r_{i}} \lambda_{i}=-\frac{1}{F_{p p}\left(\lambda_{i}\right)} \sum_{k=1, k \neq i}^{n} \frac{1}{\lambda_{i}-\lambda_{k}}$,

(b) $\partial_{r_{i}} \lambda_{j}=-\frac{1}{F_{p p}\left(\lambda_{i}\right)} \frac{1}{\lambda_{j}-\lambda_{i}}, \quad i \neq j$,

(c) $F_{p p}\left(\lambda_{i}\right) u_{r_{i}}=1$,

(d) $u_{r_{i} r_{k}}=\frac{2 u_{r_{i}} u_{r_{k}}}{\left(\lambda_{i}-\lambda_{k}\right)^{2}}$.

Remark 1. The condition (d) is in fact the so called Gibbons Tsarev compatibility condition (see [9]) (note there is a missprint in their formula-the factor 2 is missing). I did not find however the formula (a) in any paper on the subject. We suggest a very brief proof of all of them in Sect. 6 .

Corollary 2.2. In the strictly hyperbolic region $\lambda_{1}<\lambda_{2}<\cdots<\lambda_{n}$ the maximal and minimal eigenvalues are genuinely nonlinear in the sense of Lax:

$$
\partial_{r_{1}} \lambda_{1} \neq 0, \partial_{r_{n}} \lambda_{n} \neq 0 \text {. }
$$

Another important consequence of the lemma is the following:

Corollary 2.3. In the Hyperbolic region introduce the functions

$$
G_{i}=-\frac{1}{2} \log \left|u_{r_{i}}\right|=\frac{1}{2} \log \left|F_{p p}\left(\lambda_{i}\right)\right|
$$

Then it follows from the lemma that

$$
\partial_{r_{j}} G_{i}=-\frac{u_{r_{i} r_{j}}}{2 u_{r_{i}}}=-\frac{u_{r_{j}}}{\left(\lambda_{i}-\lambda_{j}\right)^{2}}=\frac{\left(\lambda_{i}\right)_{r_{j}}}{\lambda_{i}-\lambda_{j}} .
$$


To perform the blow up analysis for our system we shall differentiate the quantities $w_{i}, w_{i}=\left(r_{i}\right)_{q}$ along the integral curves of the family $\lambda_{i}$. They are, by definition, the integral curves of the equation $\dot{q}+\lambda_{i}(q, t)=0$ on $\mathbb{T}^{2}$. Let $v_{i}=\left(1, \lambda_{i}(q, t)\right)$ be the $i$ th vector field and let $L_{v_{i}}=\partial_{t}+\lambda_{i} \partial_{q}$ denote the Lie derivative along the field $v_{i}$. Differentiating with respect to $q$ the $i$ th equation of (3) one gets the following:

$$
L_{v_{i}}\left(w_{i}\right)+w_{i}^{2}\left(\lambda_{i}\right)_{r_{i}}+w_{i} \sum_{j \neq i}\left(\lambda_{i}\right)_{r_{j}}\left(r_{j}\right)_{q}=0 .
$$

Notice that by definition:

$$
L_{v_{i}} r_{j}=\left(r_{j}\right)_{t}+\lambda_{i}\left(r_{j}\right)_{q}
$$

Subtracting from this expression the $j$ th equation of (3)

$$
0=\left(r_{j}\right)_{t}+\lambda_{j}\left(r_{j}\right)_{q},
$$

one verifies that

$$
\left(r_{j}\right)_{q}=\frac{L_{v_{i}} r_{j}}{\lambda_{i}-\lambda_{j}}
$$

Substitution of this expression into (4) leads to:

$$
L_{v_{i}}\left(w_{i}\right)+w_{i}^{2}\left(\lambda_{i}\right)_{r_{i}}+w_{i} \sum_{j \neq i}\left(\lambda_{i}\right)_{r_{j}} \frac{L_{v_{i}} r_{j}}{\lambda_{i}-\lambda_{j}}=0 .
$$

Therefore it follows from the last (Corollary 2.3) that (5) can be rewritten in the following way:

$$
L_{v_{i}}\left(w_{i}\right)+w_{i}^{2}\left(\lambda_{i}\right)_{r_{i}}+w_{i} \sum_{j \neq i}\left(G_{i}\right)_{r_{j}} L_{v_{i}} r_{j}=0 .
$$

Therefore, taking into account the $i$ th equation of (3) we get:

$$
L_{v_{i}}\left(w_{i}\right)+w_{i}^{2}\left(\lambda_{i}\right)_{r_{i}}+w_{i} L_{v_{i}} G_{i}=0 .
$$

Multiplying this equation by $\exp G_{i}$ one gets rid of the linear term as follows:

$$
L_{v_{i}}\left(\left(\exp G_{i}\right)\left(w_{i}\right)\right)+\left(\exp \left(-G_{i}\right)\left(\lambda_{i}\right)_{r_{i}}\right)\left(\exp \left(2 G_{i}\right) w_{i}^{2}\right)=0 .
$$

Using the explicit expression for $G_{i}$ of Corollary 2.3 and denoting

$$
z_{i}=\left|F_{p p}\left(\lambda_{i}\right)\right|^{1 / 2} w_{i}=\left|F_{p p}\left(\lambda_{i}\right)\right|^{1 / 2}\left(r_{i}\right)_{q}, \quad K_{i}=\left|F_{p p}\left(\lambda_{i}\right)\right|^{-1 / 2}\left(\lambda_{i}\right)_{r_{i}},
$$

we get the following equation, which is crucial for the analysis of the blow up of the solution:

$$
L_{v_{i}} z_{i}+K_{i} z_{i}^{2}=0, \quad i=1, \ldots, n .
$$

As an immediate consequence of this equation we state the following:

Theorem 2.4. Let $U=\left(u_{1}, \ldots, u_{n}\right)^{t}$ be a periodic solution of the quasi-linear system (2) corresponding to the strictly Hyperbolic regime, i.e. all eigenvalues are real and distinct: $\lambda_{1}<\lambda_{2}<\cdots<\lambda_{n}$. Then the Riemann invariants $r_{1}$ and $r_{n}$ corresponding to the minimal and maximal eigenvalues are constants. 
Proof. This fact follows immediately from Eq. (8). Indeed, in the region of strict hyperbolicity the $F_{p p}\left(\lambda_{i}\right)$ does not vanish and so, by the genuine non-linearity of $\lambda_{1}$ and $\lambda_{n}$ the functions $K_{1}, K_{n}$ are bounded away from zero. Then it follows from the explicit formula for the solutions of (8) that the only solution which does not explode in finite time is $z_{1}, z_{n}=0$. Thus $\left(r_{1}\right)_{q}=\left(r_{n}\right)_{q}=0$ and Eq. (3) imply that $r_{1}$ and $r_{n}$ must be constants. This yields the result.

A refinement of this argument is the content of the next section on the Hyperbolic region, for a 3 by 3 system. In what follows we shall assume that $n=3$, i.e. the system is 3 by 3 . Let me denote by $\Omega_{h}$ the region of strict Hyperbolicity and $\Omega_{e}$ the region, where there are two complex conjugate eigenvalues for $A(U)$. The complement, $\mathbb{T}^{2}-\left(\Omega_{h} \cup \Omega_{e}\right)$ is the set of those points $(q, t)$ where the matrix $A(U)$ has at least two equal eigenvalues. We shall denote this set $\Omega_{0}$. And finally, $\Omega_{00}$ will denote the set of maximal degeneration, i.e. where all three eigenvalues are equal and thus equal to zero (since the sum of all the three eigenvalues vanishes).

\section{Hyperbolic region $\Omega_{h}$}

Before stating the main result of this section, let me rewrite the formulas of Lemma 2.1 for the case $n=3$.

In this case since $\lambda_{1}+\lambda_{2}+\lambda_{3}=0$ we get the following simplifications:

$$
\left(\lambda_{i}\right)_{r_{i}}=-\frac{3 \lambda_{i}}{F_{p p}\left(\lambda_{i}\right)^{2}}, \quad K_{i}=-\frac{3 \lambda_{i}}{\left|F_{p p}\left(\lambda_{i}\right)\right|^{5 / 2}}, \quad F_{p p}\left(\lambda_{i}\right)=\prod_{j \neq i}\left(\lambda_{i}-\lambda_{j}\right)
$$

For the case $n=3$ we have the following refinement of the theorem of the previous section.

Theorem 3.1. Let $U=\left(u_{1}, u_{2}, u_{3}\right)^{t}$ be a periodic solution of the system (2), and let $\Omega_{h} \subseteq \mathbb{T}^{2}$ be the domain of strict Hyperbolicity. Then the Riemann invariants $r_{1}, r_{3}$ are constants in every connected component of the Hyperbolic domain $\Omega_{h}$.

Proof. We give the proof for $r_{1}$, the other case is analogous. The first step of the proof is the fact that the derivatives $\left(r_{i}\right)_{q}$ are bounded functions on the domain $\Omega_{h}$. Indeed, by definition,

$$
r_{i}=F\left(\lambda_{i}, q, t\right) \Rightarrow\left(r_{i}\right)_{q}=F_{q}\left(\lambda_{i}, q, t\right)
$$

and thus by the periodicity of the coefficients of the polynomial $F$, all roots $\lambda_{i}$ of the derivative $F_{p}$ are bounded and so are the derivatives $\left(r_{i}\right)_{q}$. Consider the integral curves of the $\lambda_{1}$-family in the domain $\Omega_{h}$. Suppose that such a curve approaches the boundary of $\Omega_{h}$, then $F_{p p} \rightarrow 0$ while $\left(r_{i}\right)_{q}$ stays bounded. Therefore, it follows that

$$
z_{i}=\left(r_{i}\right)_{q}\left|F_{p p}\left(\lambda_{i}\right)\right|^{1 / 2} \rightarrow 0 .
$$

I claim that then $z_{1}$ equals zero identically in $\Omega_{h}$. If, for example, $z_{1}$ is positive at a point $\left(q_{0}, t_{0}\right)$ then by Eq. (8) and the fact that $K_{1}$ is positive, we have that $z_{1}$ is a decreasing function of time and thus in backward time along the integral 
curve cannot approach the boundary, because $z_{1}$ vanishes on the boundary. On the other hand, if the integral curve stays always inside $\Omega_{h}$ in backward time, then the function $K_{1}$ stays bounded away from zero, and therefore the blow up of the solution must occur in finite (backward) time. Thus $z_{1}$ can not be positive. The opposite case, when $z_{1}\left(q_{0}, t_{0}\right)$ cannot be negative is completely analogous.

This argument in a more precise form looks as follows. Denote by $M_{1}, M_{2}$ positive constants such that

$$
\left|\left(r_{1}\right)_{q}\right|<M_{1}, \quad \sqrt{\left|F_{p p}\left(\lambda_{i}\right)\right|}<M_{2} .
$$

Then for any backward time along the integral curve the monotonicity of $z_{1}$ implies that

$$
M_{1} \sqrt{\left|F_{p p}\left(\lambda_{1}\right)\right|}>\left(r_{1}\right)_{q} \sqrt{\left|F_{p p}\left(\lambda_{1}\right)\right|}=z_{1} \geq z_{1}\left(q_{0}, t_{0}\right)
$$

and therefore

$$
\sqrt{\left|F_{p p}\left(\lambda_{1}\right)\right|}>\frac{z_{1}\left(q_{0}, t_{0}\right)}{M_{1}}
$$

In addition, $\lambda_{1}$ cannot be too close to zero. Indeed, if $0 \leq-\lambda_{1}<a$, then by the zero sum condition also $\left|\lambda_{2}\right|<a$ and $0 \leq \lambda_{3}<2 a$. Then one would get

$$
\sqrt{\left|F_{p p}\left(\lambda_{i}\right)\right|}=\left(\left(\lambda_{2}-\lambda_{1}\right)\left(\lambda_{3}-\lambda_{1}\right)\right)^{1 / 2}<\sqrt{6} a .
$$

So together with the previous estimate this implies that $\left|\lambda_{1}\right| \geq \frac{z_{1}\left(q_{0}, t_{0}\right)}{\sqrt{6} M_{1}}$. Then

$$
K_{1}=\frac{3\left|\lambda_{1}\right|}{\left(\left(\lambda_{2}-\lambda_{1}\right)\left(\lambda_{3}-\lambda_{1}\right)\right)^{5 / 2}} \geq \frac{3 z_{1}\left(q_{0}, t_{0}\right)}{\sqrt{6} M_{1} M_{2}^{5}}>0 .
$$

So $K_{1}$ is bounded away from zero, and again by the explicit formula for the solution of (8) it explodes in finite backward time. This proves the claim that $z_{1}$ vanishes identically in $\Omega_{h}$. Thus $z_{1},\left(r_{1}\right)_{q} \equiv 0$, and so by Eq. (3) $r_{1}$ must be constant.

The next theorem describes completely the solutions of the system in the Hyperbolic region $\Omega_{h}$.

Theorem 3.2. Either the solution $U=\left(u_{1}, u_{2}, u_{3}\right)^{t}$ is a constant solution for (2) on $\mathbb{T}^{2}$, or the region $\Omega_{h}$ is a union of strips on the torus parallel to the t-axes and the following relations hold:

$$
u_{1}=u_{1}(x), \quad u_{2} \equiv 0, \quad u_{3}=u_{1}^{2}+\text { const }
$$

so that the polynomial $F$ equals (up to a constant) in $\Omega_{h}$ to the square of the Hamiltonian

$$
F=\left(\frac{p^{2}}{2}+u_{1}(q)\right)^{2}+\text { const }
$$


Proof. Let me note, that since $r_{i}$ are successive critical values of the polynomial $F_{p}$, then everywhere in $\Omega_{h} r_{2}>r_{1}, r_{2}>r_{3}$ holds. On the boundary $\partial \Omega_{h}$ two of the eigenvalues collide, say $\lambda_{2}$ collides with $\lambda_{1}$ (or with $\lambda_{3}$, or maybe both), and hence $r_{2}=r_{1}$ (or $r_{2}=r_{3}$ ). By the previous theorem $r_{1}, r_{3}$ are constants in $\Omega_{h}$, and in addition $r_{2}$ has constant values along the integral curves of the $\lambda_{2}$-family. Then it follows, that none of these curves can approach the boundary, because otherwise this would give $r_{1}=r_{2}$ in the inner points. Moreover, the function $r_{2}$ satisfies the equation

$$
\left(r_{2}\right)_{t}+\lambda_{2}\left(r_{2}\right)\left(r_{2}\right)_{q}=0,
$$

where $\lambda_{2}\left(r_{2}\right)$ depends only on $r_{2}$, since $r_{1}, r_{3}$ are constants in $\Omega_{h}$. Therefore, the characteristics of this equation are the straight lines in the $(q, t)$-plane, and thus there are two possible cases. The first case is that there exist two intersecting straight lines of the family. Then $r_{2}$ has to be constant everywhere (since $r_{2}$ has constant values along characteristics, and any straight line intersects at least one of the two intersecting characteristics). So in this case all $r_{1}, r_{2}, r_{3}$ are constants everywhere in $\Omega_{h}$, and hence also the coefficients of the polynomial $u_{1}, u_{2}, u_{3}$ are constants. In such a case $\Omega_{h}$ must be the whole of $\mathbb{T}^{2}$.

In the second case all the characteristic straight lines are parallel with the same $\lambda_{2}=\mu=$ const, and thus the domain $\Omega_{h}$ in this case is a union of parallel strips with the slope $\mu$. Next we claim that if the solution $U=\left(u_{1}, u_{2}, u_{3}\right)^{t}$ is not constant in $\Omega_{h}$, then $\mu=0$. To see this, let me recall that

$$
F_{p}(\mu)=\mu^{3}+2 u_{1} \mu+u_{2}=0,
$$

and therefore

$$
u_{2}=-2 \mu u_{1}-\mu^{3} .
$$

Substituting (9) into the first equation of the system (2) we have

$$
\left(u_{1}\right)_{t}=-\left(u_{2}\right)_{q}=2 \mu\left(u_{1}\right)_{q} .
$$

In addition we have that along any characteristic straight line of the family $\lambda_{2}$ the values of $r_{1}, r_{2}, r_{3}$ are constants, and then also $\lambda_{1}, \lambda_{2}, \lambda_{3}, u_{1}, u_{2}, u_{3}$, because $r_{1}, r_{2}, r_{3}$ are genuine coordinates. In order to prove the claim let us assume that on the contrary $\mu \neq 0$. Then $u_{1}$ has to be globally constant in $\Omega_{h}$ because it satisfies the following two equations:

$$
\left(u_{1}\right)_{t}-2 \mu\left(u_{1}\right)_{q}=0, \quad\left(u_{1}\right)_{t}+\mu\left(u_{1}\right)_{q}=0 .
$$

But then, by (9), also $u_{2}$ is constant in $\Omega_{h}$. Therefore $\lambda_{1}, \lambda_{2}, \lambda_{3}$ are all constants, since the polynomial $F_{p}$ has constant coefficients. Also since $u_{3}=r_{1}-$ $F\left(\lambda_{1}\right)$ then also $u_{3}$ must be a constant. So the solution is in fact a constant solution contradicting the assumption of the claim.

Thus, we get that $\mu=0$. In this case all characteristics of the second family are parallel to the $t$-axes and the region $\Omega_{h}$ is the union of strips parallel to the $t$-axes. Moreover by (10) $u_{1}, u_{3}$ have to be the functions on $q$ only and by (9) $u_{2} \equiv 0$. 
Therefore $F_{p}=p\left(p^{2}+2 u_{1}\right)$, and so

$$
F=\frac{1}{4} p^{4}+u_{1} p^{2}+u_{3}=\left(\frac{p^{2}}{2}+u_{1}\right)^{2}+u_{3}-u_{1}^{2},
$$

and since $r_{1}, r_{3}$ are constants, then $u_{3}-u_{1}^{2}=$ constant, and we are done. Notice that on the boudary of $\Omega_{h}, u_{1}$ vanishes and so $\partial \Omega_{h} \subseteq \Omega_{00}$.

\section{Elliptic region $\Omega_{e}$}

Consider now the region $\Omega_{e}$ where the polynomial $F_{p}$ has two complex conjugate roots, say $\lambda_{1,2}=\alpha \pm i \beta$ with $\beta>0$ in $\Omega_{e}$, and $\lambda_{3} \in \mathbb{R}$. In this case $r_{1,2}$ are also complex conjugate, say $r_{1,2}=v \pm i w$ and $r_{3}$ is real. Notice, that for the points of the boundary of $\Omega_{e}$ we have $\lambda_{1}=\lambda_{2}=-\lambda_{3} / 2$ are real, $r_{1}=r_{2}$ are real also, and so $\beta=0, w=0$. For the region $\Omega_{e}$ we have the same description of solutions as in the strictly Hyperbolic domain, but for completely different reasons.

Theorem 4.1. Either $U=\left(u_{1}, u_{2}, u_{3}\right)^{t}$ is a constant solution for the system (2) on the whole $\mathbb{T}^{2}$, or the region $\Omega_{e}$ is a union of strips parallel to the $t$-axes on the torus and and the following relations hold:

$$
u_{1}=u_{1}(q), \quad u_{2} \equiv 0, \quad u_{3}=u_{1}^{2}+\text { const } .
$$

So the polynomial $F$ equals (up to a constant) in $\Omega_{e}$ to the square of the Hamiltonian

$$
F=\left(\frac{p^{2}}{2}+u_{1}(q)\right)^{2}+\text { const } .
$$

Proof. The Riemann invariants $r_{1,2}=v \pm i w$ satisfy Eq. (3), which are equivalent to the following elliptic system on their real and imaginary parts:

$$
\begin{aligned}
& v_{t}+\alpha v_{q}-\beta w_{q}=0 \\
& w_{t}+\beta v_{q}+\alpha w_{q}=0
\end{aligned}
$$

This is an elliptic system, and therefore, since $w$ vanishes on the boundary, then by the strong maximum principle the function $w$ must vanish identically in the whole of $\Omega_{e}$. Substituting back to the elliptic system we get that $v$ is a constant everywhere in $\Omega_{e}$. Therefore, $\lambda_{1,2}$ are roots of the polynomials $F-v$ and of $F_{p}$. Then,

$$
\left.F=\frac{1}{4}\left(p-\lambda_{1}\right)^{2}\left(p-\lambda_{2}\right)^{2}+v=\frac{1}{4}\left((p-\alpha)^{2}+\beta^{2}\right)\right)^{2}+v .
$$

Moreover, $\alpha$ vanishes because $F$ does not contain the cubic terms. Therefore

$$
F=\left(p^{2} / 2+\beta^{2} / 2\right)^{2}+v, \quad \lambda_{1,2}= \pm i \beta, \quad \lambda_{3}=0 .
$$


This situation can be completely analyzed, because in this case the quadratic function $\tilde{F}=p^{2} / 2+\beta^{2} / 2$ is the conserved quantity. But then, by Eq. (1) for $\tilde{F}$ we have

$$
\begin{aligned}
\left(\beta^{2}\right)_{t} & =0, \\
\left(\beta^{2} / 2-u_{1}\right)_{q} & =0 .
\end{aligned}
$$

Notice, that on the boundary of $\Omega_{e}$ we have $\beta=u_{1}=u_{2} \equiv 0$. By the first equation, $\beta$ vanishes on any line $q=$ const whenever it crosses the boundary, and it is a constant on any line which lies entirely inside $\Omega_{e}$. This yields immediately that $\Omega_{e}$ is in fact the union of strips parallel to the $t$ axes and $\beta=\beta(q)$ and also $\beta^{2} / 2-u_{1} \equiv 0$ because on the boundary both $\beta$ and $u_{1}$ vanish. So we proved

$$
F=\left(p^{2} / 2+u_{1}(q)\right)^{2}+\text { const }
$$

and this completes the proof.

\section{Degenerate regions $\Omega_{0}, \Omega_{00}$}

It follows from the description of $\Omega_{e}$ and $\Omega_{h}$ that the degenerate region $\Omega_{0}$ is a union of strips parallel to the $t$-axes and moreover every point of the boundary of each strip belongs, in fact to $\Omega_{00}$. Then, it follows that $\Omega_{0}-\Omega_{00}$ is an open set. We claim next that the degeneration is maximal everywhere, i.e. $\Omega_{0}=\Omega_{00}$. Indeed, consider an integral curve of the $\lambda_{3}$-family lying inside this open set $\Omega_{0}-\Omega_{00}$. Then $r_{3}$ has constant values along the curve, and therefore it cannot approach the boundary of the region $\Omega_{0}-\Omega_{00}$, since otherwise one would get $r_{1}=r_{2}=r_{3}$ inside the region, which contradicts the assumptions. Thus the whole characteristic stays inside the region $\Omega_{0}-\Omega_{00}$. But then, exactly as above, in the proof of Theorem 3.1, we have that the derivative $\left(r_{3}\right)_{q}$ must explode in finite time unless it vanishes. And therefore, in the whole region $\Omega_{0}-\Omega_{00}, r_{3}$ is a constant and therefore $\Omega_{0}=\Omega_{00}$, and we are done.

\section{Proof of the Lemma 2.1}

It is rather simple to prove (a) and (b) of the lemma. By the definitions of $\lambda_{i}$ and $r_{i}$ we have

$$
F_{p}\left(\lambda_{i}\right)=0, \quad F\left(\lambda_{i}\right)=r_{i} .
$$

Differentiate these two formulas with respect to $r_{j}$. Notice that the roots $\lambda_{i}$ depend on $r_{j}$ and also the coefficients $u_{i}$ of the polynomial $F$. We get

$$
F_{p p}\left(\lambda_{i}\right)\left(\lambda_{i}\right)_{r_{j}}+F_{p r_{j}}\left(\lambda_{i}\right)=0, \quad F_{r_{j}}\left(\lambda_{i}\right)=\delta_{i j} .
$$

Then write the following identity:

$$
F_{r_{i}}(p)\left(p-\lambda_{i}\right)=\left(u_{1}\right)_{r_{i}} F_{p}(p)
$$


which becomes clear, if one notices that on both sides there are polynomials of the same degree $n$ with the same leading coefficient $\left(u_{1}\right)_{r_{i}}$, and both having $\lambda_{1}, \ldots, \lambda_{n}$ as roots [the right-hand side just by definition, and the left-hand side by (11)].

Differentiate this identity with respect to $p$ to obtain

$$
\left(p-\lambda_{i}\right) F_{p r_{i}}+F_{r_{i}}=\left(u_{1}\right)_{r_{i}} F_{p p} .
$$

Substituting $p=\lambda_{i}$ in this formula one arrives immediately at (c) of the lemma. Substitute $p=\lambda_{j}$ and take into account(11), then one proves (b).

In order to prove (a) let me use the relation $F_{r_{j}}\left(\lambda_{i}\right)=\delta_{i j}$ of (11) in order to conclude, that $F_{r_{i}}$ is, in fact, identical to the $i$ th Lagrange interpolation polynomial:

$$
F_{r_{i}}=\prod_{s \neq i} \frac{\left(p-\lambda_{s}\right)}{\left(\lambda_{i}-\lambda_{s}\right)}=l_{i}(p)
$$

Differentiate this identity with respect to $p$ at the point $\lambda_{i}$ :

$$
F_{p r_{i}}\left(\lambda_{i}\right)=\left(l_{i}\right)_{p}\left(\lambda_{i}\right)=\sum_{s \neq i} \frac{1}{\left(\lambda_{i}-\lambda_{s}\right)} .
$$

Using (11) we obtain

$$
\left(\lambda_{i}\right)_{r_{i}}=-\frac{F_{p r_{i}}\left(\lambda_{i}\right)}{F_{p p}}=-\frac{1}{F_{p p}\left(\lambda_{i}\right)} \sum_{s \neq i} \frac{1}{\left(\lambda_{i}-\lambda_{s}\right)} .
$$

This gives the proof of (a).

In order to derive (d) write (b) in the form

$$
\left(\lambda_{j}\right)_{r_{i}}=u_{r_{i}} /\left(\lambda_{i}-\lambda_{j}\right) .
$$

Following [9] differentiate this formula with respect to $r_{k}$ to get

$$
\left(\lambda_{j}\right)_{r_{i} r_{k}}=\frac{u_{r_{i} r_{k}}}{\left(\lambda_{i}-\lambda_{j}\right)}-\frac{u_{r_{i}} u_{r_{k}}}{\left(\lambda_{i}-\lambda_{j}\right)\left(\lambda_{k}-\lambda_{i}\right)\left(\lambda_{k}-\lambda_{j}\right)} .
$$

Now change the order of the indices $i$ and $k$ in (13) to have

$$
\left(\lambda_{j}\right)_{r_{k} r_{i}}=\frac{u_{r_{k} r_{i}}}{\left(\lambda_{k}-\lambda_{j}\right)}-\frac{u_{r_{k}} u_{r_{i}}}{\left(\lambda_{k}-\lambda_{j}\right)\left(\lambda_{i}-\lambda_{k}\right)\left(\lambda_{i}-\lambda_{j}\right)} .
$$

Subtract now (13) from (14). One gets (d) of the lemma.

\section{Concluding remarks and questions}

1. It would be very interesting to generalize the analysis presented here for $n=3$ to the case of higher orders. For systems of higher order, there are many more possibilities for degenerations of eigenvalues. This makes the analysis much harder. However, the case of strictly Hyperbolic solutions seems to be tractable. 
2. Besides the question of periodic solutions for the quasi-linear system, there is an interesting question to understand the global existence of smooth solutions (not necessarily periodic in time), having Hyperbolic initial data. In other words, assume that we are given for $t=0$ the initial condition, say periodic functions in $q U_{0}(q)=\left(u_{10}(q), \ldots, u_{n 0}(q)\right)$, such that the eigenvalues of the matrix $A\left(U_{0}\right)$ are real and distinct. Now switch on the dynamics. The question is whether there are solutions existing for all times, or a blow up of the solutions can be established.

3. One of the key ingredients of the proof in this paper was the observation of the richness of the quasi-linear system. It would be interesting to know what can be said in this perspective for other quasi-linear systems arising in geometry, in particular for other reductions of the Benney chain.

4. It was proved in [14] that the integration of the system (2) can be performed by the so called generalized hodograph method. However, this method is implicit and it is not clear how the proof of our main result can be obtained using this method. The main complication is that the periodic solutions in question are not known a priori to be within the regime of strict Hyperbolicity, that is the eigenvalues for the matrix $A(U)$ may collide and become complex. In this case one needs more careful analysis near the points of these collisions. Therefore, in this note we chose to follow the classical approach by Lax to catch shocks formation of the solutions especially near the boundary of the Hyperbolic region.

\section{Acknowledgments}

This paper was started during the Island 3 meeting on Integrable systems. It is my pleasure to thank the organizers and the participants. In particular I learned many facts on the Hydrodynamics type systems from Maxim Pavlov. I am pleased to take this opportunity to thank my colleagues Steven Shochet and Misha Sodin for very stimulating discussions and help. I am also thankful to Marshall Slemrod for very interesting discussions.

\section{References}

[1] Arnold, V.I., Kozlov, V.V., Neishtadt, A.I.: Mathematical aspects of classical and celestial mechanics. Dynamical systems, III, Encyclopaedia of Mathematical Sciences, vol. 3. Springer, Berlin (1993)

[2] Bialy, M.: Convex billiards and a theorem by E. Hopf. Math. Z. 214(1), 147-154 (1993)

[3] Bialy, M.: Polynomial integrals for a Hamiltonian system and breakdown of smooth solutions for quasi-linear equations. Nonlinearity 7(4), 1169-1174 (1994) 
[4] Bialy, M.: Polterovich, L. Hopf-type rigidity for Newton equations. Math. Res. Lett. 2(6), 695-700 (1995)

[5] Bialy, M.: Hamiltonian form and infinitely many conservation laws for a quasilinear system. Nonlinearity 10(4), 925-930 (1997)

[6] Delshams, A., Ramirez-Ros, R.: Poincare-Melnikov-Arnold method for analytic planar maps. Nonlinearity 9(1), 1-26 (1996)

[7] Eremenko, A.: Geometric theory of meromorphic functions. In the book: In the Tradition of Ahlfors-Bers, III, (Contemp. Math., 355), pp. 221-230. AMS, Providence, RI (2004)

[8] Gibbons, J., Kodama, Y.: Solving dispersionless Lax equations. In: Singular limits of dispersive waves (Lyon, 1991), pp. 61-66. NATO Adv. Sci. Inst. Ser. B Phys., vol. 320. Plenum, New York (1994)

[9] Gibbons, J., Tsarev, S.: Reductions of the Benney equations. Phys. Lett. A 211(1), 19-24 (1996)

[10] Lax, P.D.: Hyperbolic systems of conservation laws and the mathematical theory of shock waves. Conference Board of the Mathematical Sciences Regional Conference Series in Applied Mathematics, No. 11. Society for Industrial and Applied Mathematics, Philadelphia, PA (1973)

[11] Pavlov, M.: Classification of integrable hydrodynamic chains and generating functions of conservation laws. J. Phys. A 39(34), 10803-10819 (2006)

[12] Perelomov, A.M.: Integrable systems of classical mechanics and Lie algebras, vol. I. Birkhauser Verlag, Basel (1990)

[13] Serre, D.: Systems of conservation laws, vol. 2. Geometric structures, oscillations, and initial-boundary value problems. Translated from the 1996 French original by I. N. Sneddon. Cambridge University Press, Cambridge (2000)

[14] Tsarev, S.P.: The geometry of Hamiltonian systems of hydrodynamic type. The generalized hodograph method. (Russian) Izv. Akad. Nauk SSSR Ser. Mat. vol. 54, no. 5, pp. 1048-1068 (1990);translation in Math. USSR-Izv. vol. 37, no. 2, pp. 397-419 (1991)

M. Bialy

School of Mathematical Sciences,

Raymond and Beverly Sackler

Faculty of Exact Sciences,

Tel Aviv University,

Tel Aviv, Israel

e-mail: bialy@post.tau.ac.il

Received: 9 September 2008.

Accepted: 24 May 2009. 